\title{
BMJ Open Management of persistent postconcussion symptoms in youth: a randomised control trial protocol
}

\author{
Nick Reed, ${ }^{1,2}$ Dayna Greenspoon, ${ }^{3}$ Grant L Iverson, ${ }^{4,5,6,7}$ Carol DeMatteo, ${ }^{8,9}$ \\ Philippe Fait, ${ }^{10,11,12,13}$ Jérôme Gauvin-Lepage, ${ }^{14}$ Anne Hunt, ${ }^{1}$ \\ Isabelle J Gagnon ${ }^{15,16}$
}

To cite: Reed N,

Greenspoon D, Iverson GL, et al. Management of persistent postconcussion symptoms in youth: a randomised control trial protocol. BMJ Open 2015;5: e008468. doi:10.1136/ bmjopen-2015-008468

\section{- Prepublication history} and additional material is available. To view please visit the journal (http://dx.doi.org/ 10.1136/bmjopen-2015008468).

Received 13 April 2015 Revised 26 June 2015 Accepted 30 June 2015

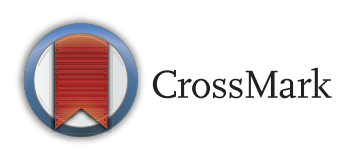

For numbered affiliations see end of article.

\section{Correspondence to}

Dr Nick Reed;

nreed@hollandbloorview.ca

\section{ABSTRACT}

Introduction: Current management of concussion consists of early education, rest until symptom free, with gradual return to school and physical activity protocols. Although this management strategy is effective for most youth who sustain a concussion, it is not an appropriate strategy for youth with persistent postconcussion symptoms. Prolonged rest and periods of restricted activity may place youth at risk for secondary issues and contribute to the chronicity of postconcussion symptoms. The purpose of this study is to evaluate the efficacy of an active rehabilitation protocol for youth who are slow to recover from concussion. It is hypothesised that an active rehabilitation intervention can reduce persistent postconcussion symptoms, improve function and facilitate return to activity. This article describes the research protocol.

Methods and analysis: This is a randomised clinical trial with blinded outcome measurement. Participants will be recruited and randomly assigned to 1 of 2 treatment groups, an active rehabilitation intervention or a standard care education group. Both groups will receive standard care education. However, the active rehabilitation group will participate in an additional low-intensity exercise programme consisting of aerobic, coordination and visualisation exercises. Both the active rehabilitation and the standard care education interventions will be 6 weeks in duration. The primary outcome measure is postconcussion symptoms. Secondary outcome measures include functional recovery (cognitive, motor, psychosocial and emotional functioning) and return to activity. Outcome measures will be administered preintervention and postintervention. The primary outcome measure will also be repeated 2 weeks into the intervention period.

Ethics and dissemination: This study has been approved by the Holland Bloorview Kids Rehabilitation Hospital research ethics board (REB \# 13-459).

The findings from this study will be shared with the general public, sport associations, relevant brain injury organisations and healthcare professionals.

Trial registration number: NCT02257749.

\section{INTRODUCTION}

According to International Consensus, concussion is defined as "A complex pathophysiological process affecting the brain, induced by biomechanical forces" (ref. 1, p.89). The acute management of concussion in children and youth includes early identification, education and reassurance, along with physical and cognitive rest. ${ }^{2}$ Return to school, often with accommodations, often occurs while the youth is symptomatic-and some clinician-researchers have provided recommendations for this. ${ }^{3-5}$ After postconcussion symptoms resolve, graduated returnto-play protocols are followed. ${ }^{2}$ International Consensus suggests that for most, recovery from a concussion is a short-lived process (80-90\% experience symptom resolution within 7-10 days). ${ }^{1}$ However, there remains a percentage of individuals who will experience persisting symptoms and will need additional care and rehabilitation. Further, it has been suggested that children and youth are more susceptible to prolonged recovery. ${ }^{1}$ An epidemiological study exploring postconcussion symptoms among children found that $58.5 \%$ of children remained symptomatic in the first month following concussion, $11 \%$ at 3 months, and $2.3 \%$ beyond 1 year. ${ }^{6}$ As a result, a significant number of youth who experience a concussion will suffer from persistent symptoms. To date, little is known about interventions to manage these youth and the research to date exploring approaches to rehabilitation specific to youth with persistent symptoms has been limited to case reports and descriptions. ${ }^{7} 8$ This study aims to address this gap by investigating two treatment approaches for youth who continue to experience symptoms lasting 1-month or longer following their concussion and applies a randomised control trial 
design to improve the methodological quality of the evidence, which might then justify improved approaches to rehabilitation within this population.

Prolonged rest and extended periods of activity restriction may place youth at risk for secondary issues (eg, physical deconditioning, irritability, anxiety, stress and depression) and may contribute to symptom maintenance over time..$^{9-11}$ A further consideration is that for many youth, absolute rest is unrealistic, and youth will naturally transition back to an active lifestyle, especially those who are only mildly symptomatic. ${ }^{9}$ Complicating these issues is that little is known about the optimal time period for rest and activity restriction following concussion. ${ }^{10}{ }^{11}$ It is possible that extended periods of rest and activity restrictions contribute to the development of secondary problems including emotional consequences. ${ }^{11}$ Unfortunately, the authors of a systematic review examining treatment for sports-related concussions ${ }^{9}$ found that the literature regarding treatment approaches is severely lacking.

Regular exercise has been shown to have positive effects on well-being including mental health, ${ }^{12}{ }^{13}$ improved self-esteem, ${ }^{14}$ sleep ${ }^{15-17}$ and memory. ${ }^{18}{ }^{19}$ It is also known to promote neuroplasticity and neurogenesis in healthy and injured brains. ${ }^{20}$ Leddy $e t a l^{21}$ used supervised exercise protocols with adults who experienced a concussion and were suffering from persistent postconcussion symptoms. Using a carefully controlled progressive programme where participants exercised on a treadmill 5-6 days per week at $80 \%$ of a predetermined symptom exacerbation threshold during exercise, participants showed significant decreases in postconcussive symptoms and recovery time. ${ }^{21}$

Recognising the promising nature of exercise as an approach to rehabilitation, Gagnon et al $l^{8}$ published a case series examining the outcome of an active rehabilitation programme on 16 children and adolescents with persistent postconcussion symptoms. The active rehabilitation programme consists of a combination of submaximal aerobic training, light sport-specific coordination exercises, visualisation and imagery techniques, and a home exercise programme. All youth had a decrease in their postconcussion symptoms, and successfully returned to their activities of daily living including sports participation following the intervention. Youth reported high satisfaction with the intervention and findings from a qualitative analysis suggest an improved sense of empowerment for youth and their parents. ${ }^{8}$ In a second case series of 10 adolescents with persistent symptoms, postconcussive symptoms and functioning improved after participation in the active rehabilitation intervention, suggesting that the introduction of graded lightintensity exercise in the postacute period following concussion is feasible and appears to have positive benefits in uncontrolled studies. ${ }^{7}$

Overall, the current literature examining exercise as a treatment modality postconcussion, and in particular, persistent postconcussion symptoms, appears positive.
However, this body of literature is very limited in quantity and quality, lacking randomisation and comparison groups. ${ }^{9}$ As such, the most recent consensus statement on concussion in sport from the 4th International Conference on Concussion in Sport Consensus Conference (2013) advocates more research on the effectiveness of these approaches in order to best meet the needs of the individuals following a concussion. This study aims to address these gaps and meet this call from the concussion clinical and research community.

The purpose of this randomised clinical trial is to examine the efficacy of an active rehabilitation protocol as compared with a standard care comprehensive education protocol for treatment of youth with persistent postconcussion symptoms (greater than 1 month postinjury). This study expands on the work of Gagnon $e t a l^{7}$ by implementing a more rigorous study methodology using a two-group, prospective, randomised control design. This study examines whether a 6 -week active rehabilitation programme in combination with standard care comprehensive education is more effective in reducing postconcussive symptoms and improving functional recovery than standard care comprehensive education alone. The study objective is to determine the efficacy of the active rehabilitation programme, compared with standard care alone, with respect to (1) reduction in postconcussion symptoms and (2) functional recovery as indicated by cognitive, motor and psychological measures. This paper presents the protocol used for this study.

\section{METHODS}

Design

This study will examine the effects of an adapted form of the active rehabilitation protocol reported by Gagnon and colleagues $^{78}{ }^{22}$ with youth who continue to experience symptoms for more than 1 month following concussion. A two-group prospective randomised design that includes blinded outcome assessment will be used. Following a telephone screening interview, participants will attend a baseline testing session and be randomised to the experimental (active rehabilitation plus standard care comprehensive education) or control group (standard care comprehensive education). Participants will be told that they are receiving one of two interventions for concussion management. Table 1 presents a summary of the study procedure. All study procedures will be conducted at a paediatric rehabilitation hospital in Toronto, Canada.

\section{Participants and recruitment}

One hundred youth with persistent symptoms for at least 1 month following concussion will be recruited. Participants will be recruited via (1) referrals from our facility's concussion clinic; and (2) flyers sent to local community practitioners (eg, family physicians, paediatricians, etc). All recruitment materials will direct interested potential participants (and their families) to contact the study coordinator to learn more. Potential 
Table 1 Summary of study procedure

\begin{tabular}{|c|c|c|c|c|}
\hline Time & Group & Session content & Method & Duration \\
\hline \multirow[t]{2}{*}{$\begin{array}{l}\text { Initial visit } \\
\text { (week 0) }\end{array}$} & $\begin{array}{l}\text { Standard care and active } \\
\text { rehabilitation groups }\end{array}$ & $\begin{array}{l}\text { Provide overview of study } \\
\text { Consent } \\
\text { Review history and confirm eligibility } \\
\text { Standard concussion education } \\
\text { Outcome measurement assessment }\end{array}$ & In-person & $1.5 \mathrm{~h}$ \\
\hline & Active rehabilitation group & $\begin{array}{l}\text { Complete the four components of the active } \\
\text { rehabilitation intervention } \\
\text { Review home training protocol and log }\end{array}$ & In-person & $1 \mathrm{~h}$ \\
\hline Week 1 & $\begin{array}{l}\text { Standard care and active } \\
\text { rehabilitation groups }\end{array}$ & $\begin{array}{l}\text { Follow-up on symptoms } \\
\text { Modify active rehabilitation programme if necessary }\end{array}$ & Phone & $10 \mathrm{~min}$ \\
\hline Week 2 & $\begin{array}{l}\text { Standard care and active } \\
\text { rehabilitation groups }\end{array}$ & Outcome measurement assessments & Phone & $10 \mathrm{~min}$ \\
\hline Week 3 & $\begin{array}{l}\text { Standard care group } \\
\text { Active rehabilitation group }\end{array}$ & $\begin{array}{l}\text { Review standard education } \\
\text { Review standard education } \\
\text { Review active rehabilitation home programme log } \\
\text { Reassessment (increase intensity of active } \\
\text { rehabilitation programme as appropriate) }\end{array}$ & $\begin{array}{l}\text { In-person } \\
\text { In-person }\end{array}$ & $\begin{array}{l}30 \mathrm{~min} \\
30 \mathrm{~min}\end{array}$ \\
\hline Week 4 & $\begin{array}{l}\text { Standard care and active } \\
\text { rehabilitation groups }\end{array}$ & $\begin{array}{l}\text { Follow-up on symptoms } \\
\text { Modify active rehabilitation programme if necessary }\end{array}$ & Phone & $10 \mathrm{~min}$ \\
\hline Week 5 & Active rehabilitation group & nues home programme & & \\
\hline Week 6 & $\begin{array}{l}\text { Standard care and active } \\
\text { rehabilitation groups }\end{array}$ & $\begin{array}{l}\text { Final outcome measurements assessments } \\
\text { - Intervention wrap-up }\end{array}$ & In-person & $2 \mathrm{~h}$ \\
\hline
\end{tabular}

participants will be screened by a telephone interview to determine eligibility. Inclusion criteria are youth between the ages of 10 and 18 years with a confirmed diagnosis of mild traumatic brain injury (mTBI) or concussion; concussive injury that occurred between 1 and 3 months prior to enrolment in the study; presence of at least one postconcussion symptom as measured by the Post-Concussion Symptom Inventory (PCSI) ${ }^{23}$ (eg, headaches, anxiety, fatigue) for greater than 4 weeks; fluent in written and spoken English and; able to demonstrate capacity to consent to participate in the study. To be enrolled, the child or adolescent must have at least one symptom that is believed to be related to the injury and has resulted in the youth not being medically cleared to fully return to sports or other recreational activities. Exclusion criteria include youth with more than one concussion in the past 6 months; presence of neck pain; presence of significant musculoskeletal pain or balance dysfunction; pre-existing comorbidities (eg, chronic pain, cardiac, mental health or autoimmune conditions) that prevent participation in active rehabilitation, assessment of gait or balance, or participation in cognitive testing; concurrent participation in other physical rehabilitation interventions for persisting postconcussion symptoms (eg, physiotherapy, massage therapy, chiropractic) at the time of enrolment and throughout the duration of the study.

\section{Informed consent}

This trial is registered with clinicaltrials.gov, registration \#: NCT02257749. An in-person meeting will be arranged with potential participants and the research coordinator to confirm study eligibility, to discuss the study procedure in greater detail, and to obtain informed written consent. At this meeting, potential participants will be provided with an information letter that outlines the study purpose, procedures and time commitment, followed by an in-person discussion with the study's research coordinator. The research coordinator will explain: (1) the purpose of the study; (2) how the randomisation process works and (3) a general overview of the two treatments and outcome measures. The discussion will also include a thorough explanation of the potential risks and benefits associated with participation in the study. The research coordinator will ensure an understanding that they can withdraw from the study at any time without question or consequence and without any effect on the care that they or their families receive. Youth and their families will be given unlimited time to consider implications, ask questions and respond to the invitation to participate in the study.

\section{Sample size}

Fifty participants will be allocated to each treatment group for a total of 100 participants. This sample size was calculated using information from other concussion studies involving high school athletes, and children who experience persistent postconcussion symptoms. ${ }^{8}$ The $\alpha$ was set at 0.01 and power was set at 0.8 . The difference between individuals receiving the active rehabilitation intervention versus the control group on the primary outcome measure, the PCSI, is expected to be six points with a pooled SD of $6.8 .^{8}$ Calculations suggest a sample 
size of 30 children per group. On the basis of previous clinical and research experience, an attrition rate of $25 \%$ is expected. As a result, this study will oversample with the aim of enrolling 50 participants per treatment group (100 participants in total).

\section{Randomisation}

Participants who meet all study inclusion criteria and consent to participate within the study will be randomly allocated to a treatment group using a stratified process. Owing to previous research indicating an increase in postconcussion symptoms reported by females, ${ }^{24}$ stratified randomisation will be used to ensure equal representation of gender across the intervention groups. A research coordinator who is not directly involved in this study will perform randomisation to ensure that the research study staff remain blinded to group allocation.

\section{Outcome measures}

All participants will be assessed at three time points throughout the duration of the study: week 0 (in-person session on enrolment in the study), week 2 (over the phone), and week 6 (in-person session on completion of the intervention). All outcome measures will be completed by a blinded assessor to minimise the risk for bias during data collection.

\section{Primary outcome}

The primary outcome of interest is postconcussion symptoms. This outcome was chosen because postconcussion symptoms are considered to be an essential clinical marker of recovery following concussion. ${ }^{1}$ Symptoms will be measured using a modified version of the PCSI. ${ }^{23}$ There are three available versions of the PCSI that will be used: (1) 21-item adolescent version, ages 13-18 years; (2) 18-item child version, ages 8-12 years and (3) 21-item parent-report scale. The PCSI captures the perceived presence and severity of symptoms following concussion. The modification is to add additional symptoms relating to sleep disturbance from the Post-Concussion Scale, ${ }^{25}$ the scale on which the PCSI was derived. Symptoms are rated on a Likert scale and are reported for before injury and after injury (current symptoms), to track changes in symptoms over brief intervals of time. PCSI has been validated for use with parents and youth following concussion. ${ }^{23}$ It has moderate to strong test-retest reliability (intraclass coefficients $=0.65-0.89$ ), moderate parent and youth concordance $(\mathrm{r}=0.44-0.65)$ and good convergent validity with a similar symptom checklist measure $(\mathrm{r}=0.8) .{ }^{23}$ It also has strong internal consistency. ${ }^{23}$ PCSI will be administered at baseline, week 2 and on completion of the intervention (week 6) for the experimental and control groups.

\section{Secondary outcomes}

Secondary measures include assessments of cognition, motor function, behaviour and emotions to evaluate functional recovery across a range of performance domains. Table 2 outlines the secondary measures used according to domain, along with the time frame within which each of the secondary measures will be administered within the study protocol.

\section{Interventions}

Control intervention: standard care (comprehensive education)

A standardised approach to delivering this concussion education and recommendations has been developed for the purposes of this study, and is reflective of the current standard care practices of the research team members representing three paediatric acute care and rehabilitation hospitals in Canada and the Ontario Neurotrauma Foundation's Guidelines for diagnosing and managing pediatric concussions. ${ }^{2}$ The components of the standard care intervention include comprehensive education about the diagnosis of concussion, self-management strategies and return to activity and school guidelines. Specific curriculum topics are outlined in box 1 .

Standard care will be provided to all participants regardless of group allocation. This education will be provided on an individual basis over the 6-week intervention period and consists of three $30 \mathrm{~min}$ in-person visits with an occupational therapist of the research team (see table 1).

\section{Experimental intervention: active rehabilitation intervention+ standard care}

In addition to the standard care (comprehensive education) described above, the experimental intervention involves an active rehabilitation programme that includes four components: (1) aerobic exercise; (2) coordination exercise; (3) visualisation exercise and (4) a home programme. This programme is based on a protocol developed and reported by Gagnon et $a l^{8}$ and has been adapted to be delivered over a period of 6 weeks. The 6 -week intervention duration was determined on the basis of findings from Gagnon $e t a l^{8}$ that symptom resolution occurred after an average of 4.4 weeks ( $\mathrm{SD}=2.6$ weeks) following the initiation of the active rehabilitation programme.

Once allocated to the experimental intervention (active rehabilitation), youth will complete a $1 \mathrm{~h}$ in-person session (week 0) that will consist of an introduction to the active rehabilitation protocol. Youth will begin by participating in an aerobic exercise of their choosing, either a fast-paced walk or light jog on a treadmill or stationary bicycle. Youth will wear a portable heart rate monitor and will be asked to exercise up to a predetermined submaximal heart rate that corresponds to $50-60 \%$ of their maximum heart rate (maximum heart rate $=220 \mathrm{bpm}$-participant age) and/or a level 4 on the Pictorial Children's Effort Rating Table (PCERT). ${ }^{37}$ Participants will continue the aerobic exercise until postconcussion symptoms are exacerbated or for a maximum of 15 min. ${ }^{8} 37$ The time to postconcussion symptom exacerbation (or $15 \mathrm{~min}$ ) becomes the time 
Table 2 Secondary measures and time frame for their completion

\begin{tabular}{|c|c|c|c|c|}
\hline \multirow[b]{2}{*}{ Domains } & \multirow[b]{2}{*}{ Measures } & \multicolumn{3}{|c|}{ Time frame for completion } \\
\hline & & $\begin{array}{l}\text { Week 0: } \\
\text { preintervention }\end{array}$ & Week 2 & $\begin{array}{l}\text { Week 6: } \\
\text { postintervention }\end{array}$ \\
\hline $\begin{array}{l}\text { Postconcussion } \\
\text { symptoms }\end{array}$ & Post-Concussion Symptom Inventory ${ }^{23}$ & $\mathrm{X}$ & $\mathrm{X}$ & $\mathrm{X}$ \\
\hline \multirow[t]{2}{*}{ Mood and anxiety } & Beck Youth Inventory-2nd Edition ${ }^{26}$ & $\mathrm{X}$ & & $\mathrm{X}$ \\
\hline & Child Behavior Checklist $^{27}$ & $\mathrm{X}$ & & $\mathrm{X}$ \\
\hline Energy level & $\begin{array}{l}\text { Pediatric Quality of Life Multidimensional Fatigue } \\
\text { Scale }^{28}\end{array}$ & $\mathrm{X}$ & & $\mathrm{X}$ \\
\hline Quality of life & Pediatric Quality of Life Generic Module ${ }^{29}$ & $\mathrm{X}$ & & $\mathrm{X}$ \\
\hline \multirow[t]{2}{*}{ Balance } & $\begin{array}{l}\text { Sport Concussion Assessment Tool-3rd Edition } \\
\text { Balance Subtest }^{1}\end{array}$ & $\mathrm{x}$ & & $\mathrm{X}$ \\
\hline & $\begin{array}{l}\text { Bruininks-Oseretsky Test of Motor Proficiency, } \\
\text { Second Edition }{ }^{30}\end{array}$ & $\mathrm{X}$ & & $\mathrm{X}$ \\
\hline Cognition & $\operatorname{ImPACT}{ }^{31}$ & $\mathrm{X}$ & & $\mathrm{X}$ \\
\hline Parental anxiety & State Trait Anxiety Scale S2 $^{32}$ & $x$ & & $x$ \\
\hline $\begin{array}{l}\text { Physical activity } \\
\text { participation }\end{array}$ & $\begin{array}{l}\text { Physical Activity Questionnaire for Children and } \\
\text { Adolescents }^{33}\end{array}$ & $\mathrm{x}$ & $\mathrm{X}$ & $\mathrm{X}$ \\
\hline $\begin{array}{l}\text { Satisfaction with } \\
\text { intervention }\end{array}$ & $\begin{array}{l}\text { Pediatric Quality of Life Health Care Satisfaction } \\
\text { Generic Module }^{34}\end{array}$ & & & $\mathrm{X}$ \\
\hline Patient characteristics & Acute Concussion Evaluation ${ }^{35}$ & $\mathrm{X}$ & & \\
\hline Goal setting & Canadian Occupational Performance Measure ${ }^{36}$ & $x$ & & $\mathrm{X}$ \\
\hline
\end{tabular}

interval for the aerobic exercise component of the participant's rehabilitation programme (take home programme and in-person visits). This time interval can be adjusted (eg, made longer up to a maximum of $15 \mathrm{~min}$ ) if the participant is able to demonstrate the ability to complete the time interval without an exacerbation of symptoms.

On completion of the aerobic training, youth will participate in light coordination exercises for up to $10 \mathrm{~min}$. These activities will be tailored to the child's favourite sport or physical activity. For example, a North American soccer player may be asked to complete a series of ball and footwork activities that are standard in typical practices. Heart rate will continue to be monitored to ensure that it does not exceed $50-60 \%$ of their maximal capacity. ${ }^{8}$ Since research has shown that youth experience decreased self-efficacy in relation to their physical activity performance after mTBI, ${ }^{38}$ these exercises combine light aerobic exercise with familiar sport activities in a monitored and supervised environment to

\section{Box 1 Standard care}

\section{Standard Care Education Topics}

-What is a concussion?

- Energy conservation

- Sleep hygiene

- Relaxation strategies

- Nutrition and hydration

- Return-to-school recommendations

- Return-to-activity recommendations

Self-management tools target self-efficacy and physical function. ${ }^{8}$ Participants will be closely monitored for symptom exacerbation during the coordination exercises. If postconcussion symptoms are exacerbated, the exercises are stopped immediately ${ }^{8}$ and procedures for symptom exacerbation will be followed (see Safety reporting and monitoring section).

Following the completion of coordination exercise, youth will engage in a sport-specific visualisation exercise. The purpose of this exercise is to reassociate positive experiences in relation to physical activity participation and to target self-confidence in return to sports and physical activities. Participants will be asked to choose a specific physical activity associated with their favourite sport for the visualisation exercise. Specifically, they will be asked to choose an activity that they typically perform successfully and that is finite in duration (eg, footwork or dribbling drills in North American soccer). ${ }^{8}$

Home programme: The final part of the active rehabilitation intervention includes a daily home programme. The home programme consists of daily practice of the aerobic training, coordination drills and visualisation exercises at the level predetermined in the initial session in the clinic and without exacerbation of symptoms. Participants will monitor their participation by recording their completion of each programme component using a daily $\log$ designed for this purpose. ${ }^{8}$ Parents will be asked to facilitate completion of the daily log. Participants will be contacted by phone at weeks 1,2 and 4 , and they will have an in-person re-evaluation at week 3 . The home programme may be modified by the therapist at any of these time points depending on the participant's symptoms and progress. A third and final 
in-person session will be held at week 6 . During this session, youth will be verbally instructed in the standard return-to-activity protocol provided they report remaining symptom free at rest for at least 1 week. Youth who continue to experience persistent symptoms beyond the 6-week intervention may be referred to the Concussion Clinic at Holland Bloorview Kids Rehabilitation Hospital for evaluation by a physician and/or rehabilitation team.

\section{Compliance}

Participants will be aware that they can withdraw from the study at any time without penalty. Those who choose to withdraw from the study, regardless of their treatment group assignment (standard care only vs active rehabilitation), will be given the opportunity to participate in the protocol's final data collection point (week 6). This is to ensure that important data (such as ceased participation due to negative outcomes) are not excluded from the analysis. An analysis of participants who complete at least $80 \%$ of the intervention will also be included for exploratory purposes.

Participants will be asked to make up any missed evaluation sessions as soon as possible (preferably within 9 days of the date the evaluation was initially scheduled). Any missed active rehabilitation and standard education sessions are required to be made up as soon as possible. The 6-week intervention can extend to a maximum of 9 weeks secondary to missed sessions. The participant is considered to have dropped out of the study if these criteria are not met.

\section{Co-intervention and contamination}

On the basis of our experience, it is expected that some children will be pursuing concurrent physical rehabilitation interventions (eg, physiotherapy, massage therapy, chiropractic) for their postconcussion symptoms. Owing to the nature of the randomised control trial design and risk for co-intervention bias, youth will be asked not to participate in any concurrent physical rehabilitation for postconcussion symptoms throughout the duration of the study. Participation in a concurrent physical rehabilitation intervention would impede the study's ability to determine the effectiveness of the active rehabilitation intervention as a treatment for postconcussion symptoms, and therefore is considered to be the exclusion criterion. A research coordinator will inquire about co-occurring treatment at weeks 0,3 and 6 to ensure that this exclusion criterion continues to be met throughout the duration of the study.

To monitor for contamination (participation in activity outside of the intervention protocol), youth's activity levels will be tracked using data collected from the Physical Activity Questionnaire for Children and Adolescents $^{33}$ (see table 2 for administration schedule).

\section{Safety reporting and monitoring}

The risk for increased fatigue and exacerbation of postconcussion symptoms will be disclosed to participants.
Although it is possible that participants may experience an exacerbation of symptoms with increased activity, there are also risks associated with prolonged inactivity, including an increase in postconcussion symptoms. ${ }^{9}$ Participants will be provided with the opportunity to take rest breaks as needed during study intervention and/or data collection sessions.

Participants will be asked to report any new symptoms, or increases in frequency or intensity of postconcussion symptoms, to the principal investigator throughout the duration of the study. The principal investigator will keep a $\log$ of all adverse events for both treatment groups to enable comparisons on completion of the study. Youth will be advised to stop the active rehabilitation intervention and rest at the onset or exacerbation of symptoms. Rest should reverse symptom exacerbation. Youth will be permitted to continue the protocol once symptoms have resolved and/or returned to baseline for at least $24 \mathrm{~h}$. If symptoms persist and/or worsen, the principal investigator will assess whether: (1) the child can continue with the intervention; (2) the intervention needs to be discontinued or (3) the intervention needs to be suspended for a specific period of time. These youth will be referred back to their community physicians as needed and may require medical clearance before resuming participation in the study.

\section{Planned analyses}

Baseline characteristics will be compared using descriptive statistics (mean, SD, 95\% CIs). Differences in patient characteristics (eg, age, gender, mechanism of injury and presenting postconcussion symptoms) between the experimental and control groups will be examined using Student t tests (Wilcoxon rank-sum tests are used if the assumptions of normality and homogeneity of variance are not met) and $\chi^{2}$ tests. Any significant difference between groups will be included as covariates in further analyses. Analyses will be based on intention to treat. To address aim 1, comparing changes in postconcussion symptoms (PCSI) postintervention, a two-way analysis of variance (ANOVA) (group $\times$ time (baseline, 2 weeks, 6 weeks) with repeated measures on the time factor will be performed (Friedman two-way ANOVA by ranks will be used if the assumptions of normality and homogeneity of variance are not met). The 6-week interval will be of primary interest. Similar analyses with the secondary outcome measures will be performed for the baseline to 6 -week data to address aim 2 .

\section{Dissemination}

Knowledge generated from this study will be shared with healthcare professionals, the general public, sport associations, and brain injury and relevant organisations, through publication of manuscripts, conference presentations and seminars/rounds. Further, this study will engage knowledge translation specialists to create an implementation strategy to reach as many stakeholders and members of the youth sport community as possible. 
CONCLUSION: IMPACT ON CLINICAL PRACTICE

This article describes a protocol for a randomised control trial that tests the efficacy of two interventions for the rehabilitation of youth with persistent postconcussion symptoms: active rehabilitation plus standard care (comprehensive education) and standard care only. Results from primary and secondary measures will improve our understanding of the efficacy of the two interventions in relation to physical, cognitive, socioemotional and functional recovery. Knowledge from this study will help clinicians make informed evidence-based decisions regarding return to activity following concussion.

\section{CURRENT STUDY STATUS}

This trial is set to begin recruitment in July/August 2015.

\section{Author affiliations}

${ }^{1}$ Concussion Research Centre, Bloorview Research Institute, Holland Bloorview Kids Rehabilitation Hospital, Toronto, Ontario, Canada

${ }^{2}$ Department of Occupational Science and Occupational Therapy, University of Toronto, Toronto, Ontario, Canada

${ }^{3}$ Concussion Research Centre, Centre for Leadership in ABI, Bloorview Research Institute, Holland Bloorview Kids Rehabilitation Hospital, Toronto, Ontario, Canada

${ }^{4}$ Department of Physical Medicine and Rehabilitation, Harvard Medical School, Boston, Massachusetts, USA

${ }^{5}$ Spaulding Rehabilitation Hospital, Boston, Massachusetts, USA

${ }^{6}$ MassGeneral Hospital for Children Sports Concussion Program, Boston,

Massachusetts, USA

${ }^{7}$ Red Sox Foundation and Massachusetts General Hospital Home Base

Program, Boston, Massachusetts, USA

${ }^{8}$ School of Rehabilitation Science, McMaster University, Hamilton, Ontario, Canada

${ }^{9}$ CanChild Centre for Childhood Disability Research, Hamilton, Ontario, Canada

${ }^{10}$ Department of Human Kinetics Sciences, Université du Québec à TroisRivières (UQTR), Trois-Rivières, Quebec, Canada

${ }^{11}$ Research Group on Neuromusculoskeletal Dysfunctions, UQTR, TroisRivières, Quebec, Canada

${ }^{12}$ Centre de Recherche en Neuropsychologie, Montreal, Quebec, Canada

${ }^{13}$ Clinique Cortex Medicine et Readaptation, Quebec City, Quebec, Canada

${ }^{14}$ School of Physical and Occupational Therapy, McGill University, Montreal, Quebec, Canada

${ }^{15}$ Faculty of Medicine, School of Physical and Occupational Therapy, McGill University, Montreal, Quebec, Canada

${ }^{16}$ Trauma, Montreal Children's Hospital of the McGill University Health Center Montreal, Quebec, Canada

Twitter Follow Nick Reed at @DrNickReed and Philippe Fait at @DrPhilFait

Acknowledgements The authors acknowledge sport concussion research funding from the Mooney-Reed Charitable Foundation.

Contributors IJG designed the experimental intervention. NR, IJG, GLI, CD and $P F$ refined the study protocol and study implementation. DG coordinated the protocol manuscript development. All the authors contributed to the writing and editing of the manuscript.

Funding Funding for the pilot study informing this protocol was provided by Fonds de recherche du Québec-Santé.

Competing interests GLI has been reimbursed by the government, professional scientific bodies and commercial organisations for discussing or presenting research relating to mild TBI and sport-related concussion at meetings, scientific conferences and symposia. He has a clinical and consulting practice in forensic neuropsychology involving individuals who have sustained mild TBIs. He has received research funding from several test publishing companies, including ImPACT Applications, Inc, CNS Vital Signs and Psychological Assessment Resources (PAR, Inc). He is a co-investigator, collaborator or consultant on grants relating to mild TBI funded by several organisations, including, but not limited to, the Canadian Institute of Health Research, Alcohol Beverage Medical Research Council, Rehabilitation Research and Development (RR\&D) Service of the US Department of Veterans Affairs, Vancouver Coastal Health Research Institute and Roche Diagnostics Canada. PF is the founder and co-owner of Cortex Medicine and rehabilitation clinic.

Ethics approval Ethics approval has been received from Bloorview Research Institute and Holland Bloorview Kids Rehabilitation Hospital's Research Ethics Board.

Provenance and peer review Not commissioned; externally peer reviewed.

Open Access This is an Open Access article distributed in accordance with the Creative Commons Attribution Non Commercial (CC BY-NC 4.0) license, which permits others to distribute, remix, adapt, build upon this work noncommercially, and license their derivative works on different terms, provided the original work is properly cited and the use is non-commercial. See: http:// creativecommons.org/licenses/by-nc/4.0/

\section{REFERENCES}

1. McCrory P, Meeuwisse W, Aubry M, et al. Consensus statement on concussion in sport-the 4th International Conference on Concussion in Sport held in Zurich, November 2012. Clin J Sport Med 2013;23:89-117.

2. Zemek R, Duval S, Dematteo C, et al. Guidelines for diagnosing and managing pediatric concussions. Toronto, Ontario: Ontario Neurotrauma Foundation, 2014

3. DeMatteo C, Stazyk K, Giglia L, et al. A balanced protocol for return to school for children and youth following concussive injury. Clin Pediatr (Phila) 2015;54:783-92.

4. Halstead ME, McAvoy K, Devore CD, et al. Returning to learning following a concussion. Pediatrics 2013;132:948-57.

5. McGrath N. Supporting the student-athlete's return to the classroom after a sport-related concussion. J Athl Train 2010;45:492-8.

6. Barlow KM, Crawford S, Stevenson A, et al. Epidemiology of postconcussion syndrome in pediatric mild traumatic brain injury. Pediatrics 2010;126:e374-81.

7. Gagnon I, Grilli L, Friedman D, et al. A pilot study of active rehabilitation for adolescents who are slow to recover from sport-related concussion. Scand J Med Sci Sports 2015 Published Online First: 3 March 2015.

8. Gagnon I, Galli C, Friedman D, et al. Active rehabilitation for children who are slow to recover following sport-related concussion. Brain Inj 2009;23:956-64.

9. Schneider KJ, Iverson GL, Emery CA, et al. The effects of rest and treatment following sport-related concussion: a systematic review of the literature. Br J Sports Med 2013;47:304-7.

10. Silverberg ND, Iverson GL. Is rest after concussion "the best medicine?": recommendations for activity resumption following concussion in athletes, civilians, and military service members. $J$ Head Trauma Rehabil 2013;28:250-9.

11. Thomas DG, Apps JN, Hoffmann RG, et al. Benefits of strict rest after acute concussion: a randomized controlled trial. Pediatrics 2015;135:213-23.

12. Cooney GM, Dwan K, Greig CA, et al. Exercise for depression. In: Cochrane database of systematic reviews. John Wiley \& Sons, Ltd, 1996. http://onlinelibrary.wiley.com/doi/10.1002/14651858. CD004366.pub6/abstract (accessed 15 May 2014).

13. Mead GE, Morley W, Campbell $P$, et al. Exercise for depression. In: Cochrane database of systematic reviews. John Wiley \& Sons, Ltd, 1996. http://onlinelibrary.wiley.com/doi/10.1002/14651858. CD004366.pub4/abstract (accessed 15 May 2014).

14. Ekeland E, Heian F, Hagen KB, et al. Exercise to improve self-esteem in children and young people. Cochrane Database Syst Rev 2004;(1):CD003683.

15. Brand S, Gerber M, Beck J, et al. High exercise levels are related to favorable sleep patterns and psychological functioning in adolescents: a comparison of athletes and controls. J Adolesc Health 2010;46:133-41.

16. Brand S, Beck J, Gerber M, et al. "Football is good for your sleep": favorable sleep patterns and psychological functioning of adolescent male intense football players compared to controls. J Health Psycho 2009;14:1144-55. 
17. Youngstedt SD. Effects of exercise on sleep. Clin Sports Med 2005;24:355-65, xi.

18. Chaddock L, Erickson KI, Prakash RS, et al. A neuroimaging investigation of the association between aerobic fitness, hippocampal volume, and memory performance in preadolescent children. Brain Res 2010;1358:172-83.

19. Chaddock L, Erickson KI, Prakash RS, et al. Basal ganglia volume is associated with aerobic fitness in preadolescent children. Dev Neurosci 2010;32:249-56.

20. Griesbach GS, Hovda DA, Molteni R, et al. Voluntary exercise following traumatic brain injury: brain-derived neurotrophic factor upregulation and recovery of function. Neuroscience 2004;125:129-39.

21. Leddy JJ, Kozlowski K, Donnelly JP, et al. A preliminary study of subsymptom threshold exercise training for refractory post-concussion syndrome. Clin J Sport Med 2010;20:21-7.

22. Iverson GL, Gagnon I, Griesbach GS. Active rehabilitation for slow-to-recover children following mild traumatic brain injury. In: Yeates KO, Kirkwood M, eds. Mild traumatic brain Injury in children and adolescents: from basic science to clinical management. New York: Guilford Press, 2012:281-302.

23. Sady MD, Vaughan CG, Gioia GA. Psychometric characteristics of the postconcussion symptom inventory in children and adolescents. Arch Clin Neuropsychol 2014. Published Online First: 15 April 2014.

24. Covassin T, Elbin RJ, Harris W, et al. The role of age and sex in symptoms, neurocognitive performance, and postural stability in athletes after concussion. Am J Sports Med 2012;40:1303-12.

25. Lovell MR, Iverson GL, Collins MW, et al. Measurement of symptoms following sports-related concussion: reliability and normative data for the postconcussion scale. Appl Neuropsychol 2006;13:166-74.

26. Beck J, Beck A, Jolly J, et al. Beck youth inventories-second edition for children and adolescents BYIII. San Antonio, TX: Harcourt Assessment Inc., 2005

27. Achenback TM. Manual for Child Behavior Checklist/ 4-18 and 1991 profile. Burlington, VT: University of Vermont, Dept. of Psychiatry, 1991.
28. Varni JW, Burwinkle TM, Katz ER, et al. The PedsQL in pediatric cancer: reliability and validity of the Pediatric Quality of Life Inventory Generic Core Scales, Multidimensional Fatigue Scale, and Cancer Module. Cancer 2002;94:2090-106.

29. Varni JW, Seid M, Kurtin PS. PedsQL 4.0: reliability and validity of the Pediatric Quality of Life Inventory version 4.0 generic core scales in healthy and patient populations. Med Care 2001;39:800-12.

30. Bruininks RH, Bruininks BD. Bruininks-Oseretsky test of motor proficiency. 2nd edn. Circle Pines, MN: Pearson, 2005.

31. Maroon JC, Lovell MR, Norwig J, et al. Cerebral concussion in athletes: evaluation and neuropsychological testing. Neurosurgery 2000;47:659-69; discussion 669-72.

32. Spielberger $\mathrm{C}$. Test of anxiety inventory: preliminary professional manual. Palo Alto, CA: Consulting Psychologists Press, 1980.

33. Crocker PR, Bailey DA, Faulkner RA, et al. Measuring general levels of physical activity: preliminary evidence for the Physical Activity Questionnaire for Older Children. Med Sci Sports Exerc 1997;29:1344-9.

34. Varni JW, Burwinkle TM, Dickinson P, et al. Evaluation of the built environment at a children's convalescent hospital: development of the Pediatric Quality of Life Inventory parent and staff satisfaction measures for pediatric health care facilities. J Dev Behav Pediatr 2004;25:10-20.

35. Gioia GA, Collins M, Isquith PK. Improving identification and diagnosis of mild traumatic brain injury with evidence: psychometric support for the acute concussion evaluation. J Head Trauma Rehabil 2008;23:230-42.

36. Carswell A, McColl MA, Baptiste S, et al. The Canadian occupational performance measure: a research and clinical literature review. Can J Occup Ther 2004;71:210-22.

37. Roemmich JN, Barkley JE, Epstein LH, et al. Validity of PCERT and OMNI walk/run ratings of perceived exertion. Med Sci Sports Exerc 2006;38:1014-19.

38. Gagnon I, Swaine B, Friedman D, et al. Exploring children's self-efficacy related to physical activity performance after a mild traumatic brain injury. J Head Trauma Rehabil 2005;20:436-49. 